(C) 2016 IEEE. Personal use of this material is permitted. Permission from IEEE must be obtained for all other uses, in any current or future media, including reprinting/republishing this material for advertising or promotional purposes, creating new collective works, for resale or redistribution to servers or lists, or reuse of any copyrighted component of this work in other works. 


\title{
A Telepresence Wheelchair Using Cellular Network Infrastructure in Outdoor Environments
}

\author{
Van Kha Ly Ha, Student Member, EMBS, IEEE, Tuan Nghia Nguyen, Senior Member, IEEE, \\ and Hung T. Nguyen, Senior Member, IEEE
}

\begin{abstract}
Mobile wireless network technology has grown rapidly over the past decade with emerging applications and services. Particularly, the fourth generation (4G) cellular network has acted as a bridge between telecommunication technology and daily life applications. In this paper, we present an investigation into a telepresence wheelchair in outdoor environments employing cellular network infrastructure instead of using local wireless networks in indoor environments. Experiments were carried out to demonstrate remote interaction and control from a long distance and across countries. A large amount of communication data based on real network measurements was collected and analyzed to evaluate the system performance. The experimental results show that a wheelchair system can be controlled remotely in real-time with the acceptable round trip time of less than $400 \mathrm{~ms}$. The results reveal the feasibility of using the $4 \mathrm{G}$ network for a telepresence wheelchair in healthcare applications.
\end{abstract}

\section{INTRODUCTION}

In the modern world, innovations in telecommunications have made significant impacts on human modern life and offered numerous applications in daily life. However, the use of advanced telecommunication technologies to develop solutions for improving healthcare such as tele-monitoring, social interaction, and remote healthcare assistance through a wheelchair for people with disabilities is limited due to its technical challenges [1].

Recently, smart wheelchairs for people with disabilities have attracted considerable attention. There are a wide variety of smart wheelchairs that have been developed by researchers [2], and references therein. The focus of these studies has been on direct assistance while a wheelchair user is sitting in a wheelchair. Our work focused on the control of wheelchairs using head movement, brain waves, and sensors. One typical example [3], described an intelligent wheelchair which works as a hands-free wheelchair control system and provides obstacle detection while navigating through an unknown environment to assist wheelchair users. However, the ability to control the mobility of wheelchairs from a remote location, long distance, has not been studied in the literature thus far, although such meaningful systems are useful for people with disabilities.

Van Kha Ly Ha, Tuan Nghia Nguyen, Hung T. Nguyen are with the Centre for Health Technologies, Faculty of Engineering and Information Technology, University of Technology Sydney, NSW 2007, Australia, (email:VanKha.L.Ha@student.uts.edu.au; tuannghia.nguyen@uts.edu.au; Hung.Nguyen@uts.edu.au).
Much research has been done in the field of telepresence. Telepresence involves many technologies such as robotics, computer and telecommunication networks. Apart from telepresence robots for meetings, telepresence robots for education, and for visiting people have been explored in [4] and [5], respectively. These telepresence robots were developed through the addition of a webcam, computer, and mobile robot platform $[4,5]$. To date, current telepresence works have been limited in the field of robotic applications and being used only in indoor environments.

The advancement of telepresence technology has not stopped, though. People with disabilities would benefit from the use of these technologies. There is a gap in the literature with regard to developing a so-called telepresence wheelchair. The use of telepresence wheelchairs could provide mobility assistance to people with disabilities when distance separates the participants. This system with realtime video interaction would allow a remote user to interact, and assist a distant wheelchair user. The system not only provides healthcare applications to wheelchair users, but also acts as a bridge between them and remote users, doctors, and their relatives.

The main objective of this paper is to present our practical investigation of a telepresence wheelchair using cellular networks. Our approach implicitly exploits technological advancement in telecommunication, automation control, and healthcare systems. Our key contributions demonstrate the feasibility of a telepresence wheelchair for face-to-face communication and efficient remote control via a cellular network medium. In particular, we have developed appropriate software for two-way video interaction and telepresence wheelchair remote control based on the Skype framework. We conducted real-time experiments in a long distance connection with the function of telepresence in outdoor environments. The remote user can drive the wheelchair via the Internet. At the same time, the remote user can hear sound and see the surrounding areas of the wheelchair with the 360-degree view in real-time. The performance of such an interactive telepresence wheelchair using a 4G mobile network was considered. Technical performance measurements were conducted to collect transmission data for evaluation of the system performance.

The remainder of this paper is organized as follows. Section II describes the design of the telepresence wheelchair system, the Skype framework, and the 4G cellular network. Section III is devoted to the experiments, results, and discussion. Finally, conclusions and future works are drawn in Section IV. 


\section{METHODOLOGY}

\section{A. Design of the Telepresence Wheelchair}

The telepresence wheelchair is a system that provides realtime audio and video communication capabilities, and can be controlled remotely from a long distance. The telepresence wheelchair platform has been developed as shown in Fig. 1. In order to achieve the desired features, the powered wheelchair is equipped with a Mac mini computer, control components, telecommunication interfaces, touchscreen display and four webcams for a wide view surrounding the wheelchair. Particularly, a NI USB-6008 is connected on the back of the wheelchair to transform movement commands from the remote user to control the wheelchair. NI USB6008 is controlled by an application that can be plugged into Skype framework.

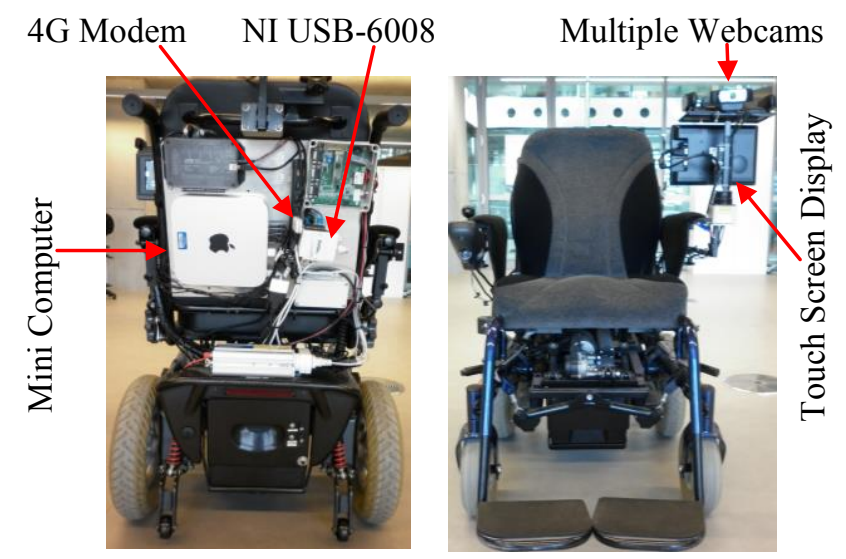

Figure 1. The back and front view of the telepresence wheelchair.

\section{B. Skype Framework}

Skype has changed the world with its free voice-over-IP service, proving high-quality two-way video communication over the Internet [6]. There are other frameworks for telepresence such as the Server and Client website, and WebRTC. However, Skype is a well-known cross-platform, since users can expand using an Application Programming Interface (API). It is an ideal framework for constructing an inexpensive system. For that reason, we have developed a telepresence wheelchair based on this ubiquitous framework. Our approach is adapted to the specific application in the field of health care.

To be able to achieve the remote interaction characteristics, a control interface with five buttons, namely go forward, turn left, turn right, go backward and stop was designed to send command signals to Skype. These control signals are written and read by an API developed from C\#. At the wheelchair side, these commands will be received and transmitted to the NI USB 6008 to drive the wheels in realtime. An application with the functions of acquiring, displaying a live video stream and controlling the wheelchair was developed. In this application, once the connection is established, a remote user will be able to view live video around the wheelchair and communicate with a wheelchair user by using the $4 \mathrm{G}$ cellular network.

\section{C. $4 G$ mobile network architecture}

The development of the cellular network has revolutionized the transfer of information. Particularly, the fourth generation cellular network (4G) or Long-Term Evolution (LTE). The LTE is a standard for wireless communication of high-speed data for mobile phones and data terminals developed by the 3rd Generation Partnership Project (3GPP) [7]. In theory, 4G increases the capacity and speed using a different radio interface together with core network improvements with the peak of download speeds up to $100 \mathrm{Mbps}$ and $50 \mathrm{Mbps}$ upload. The LTE network architecture diagram is shown in Fig. 2. It illustrates the connection paths from the end user equipment (UE) to the evolved base stations (eNodeB). Each eNodeB is a base station that communicates with and controls the mobiles. The mobility management is responsible for choosing the serving gateway (S-GW). S-GW acts as a router, and exchanges data between the base station and the packet data network gateway (P-GW), and communicates with the outside world such as the Internet or the IP networks.

The telepresence wheelchair requires good quality video streaming and stable communication links between geographically distributed users. Understanding the network performance is important for an effective telepresence system. The control processing capabilities of the wheelchair depend on the transmission delays and other factors. In particular, due to mobility issues and radio conditions, fluctuation signals and the lack of bandwidth cause latency and jitter which can impact the overall system performance. Latency or round-trip time (RTT) refers to the amount of time it takes a bit from transmission to reception. The large RTTs result in system connection time-out. Jitter causes packets to arrive at their destination with different timing. RTT and jitter calculation has been presented in [8]. It denotes the corresponding event times as $T s_{i}$ and $T r_{i}$ for sending and receiving time respectively (for packet ID =i). The $R T T_{i}$ is the difference between the event times

$$
R T T_{i}=T r_{i}-T s_{i}
$$

For jitter calculation, expression (2) is used. In this formula the individual latency measurement samples for two subsequently received packets of the same stream are defined as $R T T_{i}, R T T_{j}$ and the difference of these two latency measurement results is defined as $J(j, i)$ :

$$
\begin{aligned}
& J_{1}=0, \quad J_{2}=(2,1) \\
& J_{i}=J_{i-1}+\left.\frac{\left(|J(i, i-1)|-J_{i-1}\right)}{16}\right|_{\substack{i=3 \\
i=\infty}}
\end{aligned}
$$

The factor of 16 in the denominator of (2) counts for a smoothing factor proposed in the standard RFC 1889 [8].

In this study, a mobile broadband data $4 \mathrm{G}$ modem is plugged in the wheelchair and acts as a UE for telecommunication interfaces as shown in Fig. 1. Therefore, the telepresence wheelchair can establish connections to the Internet via the telecommunication infrastructures for transferring video and control signals. 


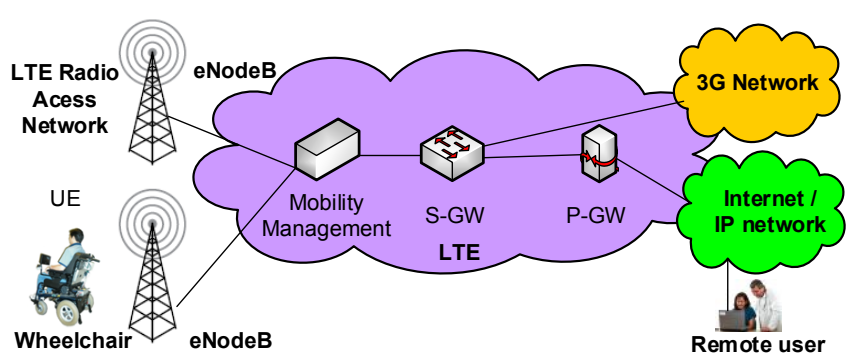

Figure 2. A simplified LTE cellular network architecture.

\section{EXPERIMENTS AND RESULTS}

\section{A. Experimental setup}

The experimental study on the performance of the telepresence wheelchair was deployed in outdoor environments. The wheelchair was located within the pedestrian walkway at Jones Street in Sydney, Australia. The system configurations of a remote user and wheelchair are listed in Table I. The wheelchair computer was configured with a 4G modem on the Telstra mobile network in Sydney, New South Wales, Australia. In this setup, a wheelchair user was required to sit in the wheelchair without doing anything and remote users connected, interacted and navigated the wheelchair as shown in Fig. 3a.

We conducted two case studies. First, we carried out experiments with domestic connections in the same country. In this scenario, remote users and a wheelchair user would be in Australia. In the second scenario, we set up other experiments to connect and control the wheelchair with the function of telepresence from a different country in a long distance connection. The remote user was in another country (Vietnam) and the wheelchair user was in Australia as shown in Figs. 3a, 3b and 4. For our system performance to be analysed and evaluated, several measurements were taken at each site during the connection to measure the impact factors for ongoing connections. Four key performance parameters were taken into account, including signal strength, round-trip time, jitter and central processor unit usage. The results were used to analyse the performance of our system and made comparisons with previous studies. Wireshark was used to capture and analyse network traffic [9]. Pings were simultaneously sent from the remote user to the wheelchair for RTT measurements, and the respective latency data was saved into a $\log$ file.

TABLE I. DETAILS OF THE COMPUTERS USED FOR IMPLEMENTATION.

\begin{tabular}{l|l|l}
\hline System information & Remote computer & \multicolumn{1}{|c}{ Wheelchair computer } \\
\hline \hline Model & MacBook pro & Mac mini \\
\hline CPU, DRAM & Intel Core i7, 8 GB & Intel Core i7, 8 GB \\
\hline OS & Window 7 (64 bit) & Window 7 (64 bit) \\
\hline Number of webcams & 1 & 4 \\
\hline Skype & Version 7.17.0.105 & Version 7.17.0.105 \\
\hline Wireless & Wi-Fi & $4 \mathrm{G}$ \\
\hline Transport protocol & TCP/IP, UDP & TCP/IP, UDP \\
\hline Movement speed & 0 & $1 \mathrm{~m} / \mathrm{s}$ \\
\hline
\end{tabular}

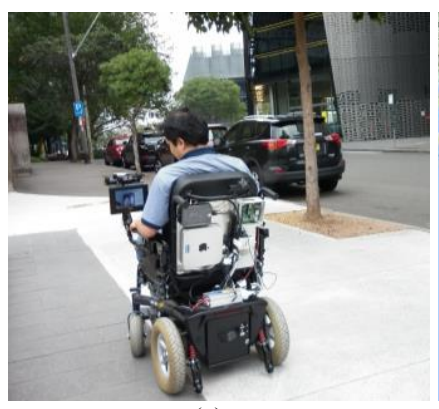

(a)

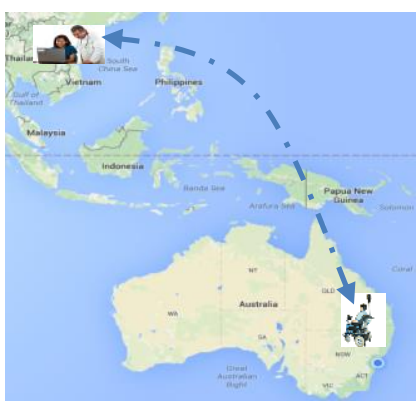

(b)
Figure 3. (a) Wheelchair in outdoor environment at Jones street - Sydney. (b) A connection from remote user in Vietnam to wheelchair user in Sydney

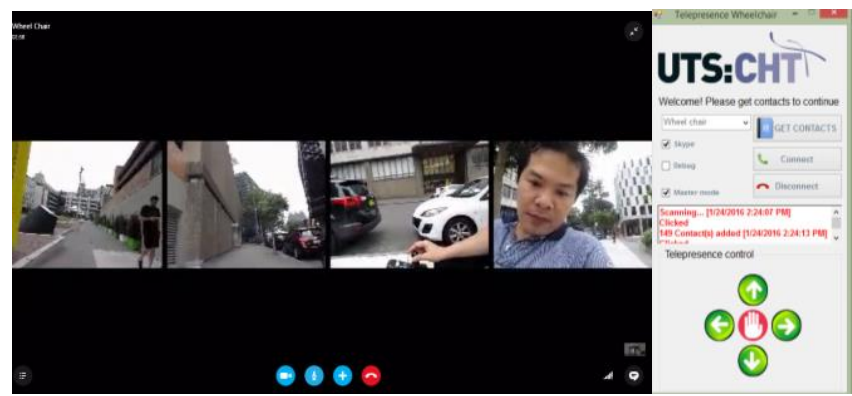

Figure 4. Remote user controlled wheelchair from a long distance.

\section{B. Results and Discussion}

Radio reception strength of the $4 \mathrm{G}$ modem on the computer of the wheelchair was measured in reference signal received power (RSRP) in decibel milliwatt $(\mathrm{dBm})$ at a different time while the wheelchair was moving. The measurement results are described in Fig. 5. RSRP varied between -93 and $-101 \mathrm{dBm}$ for the first scenario and -93 and $-107 \mathrm{dBm}$ for the second scenario. In comparison with [10], the mobile reception signal of the wheelchair was sufficient for a remote user to connect to the wheelchair and control the wheelchair with telepresence functions.

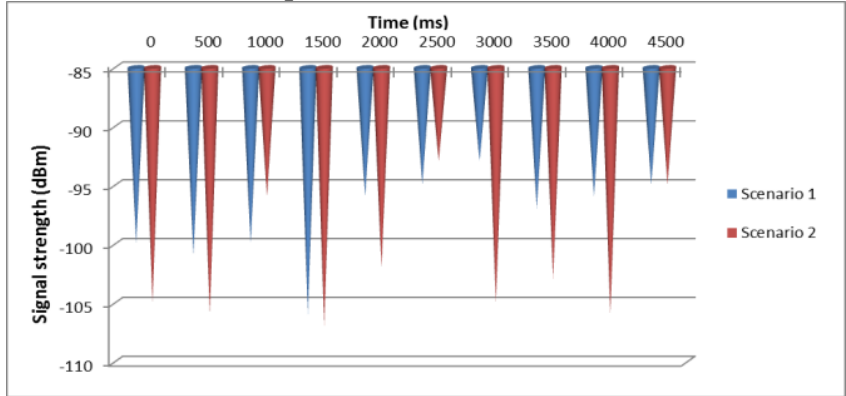

Figure 5. The RSRP measurement from the wheelchair of experiments

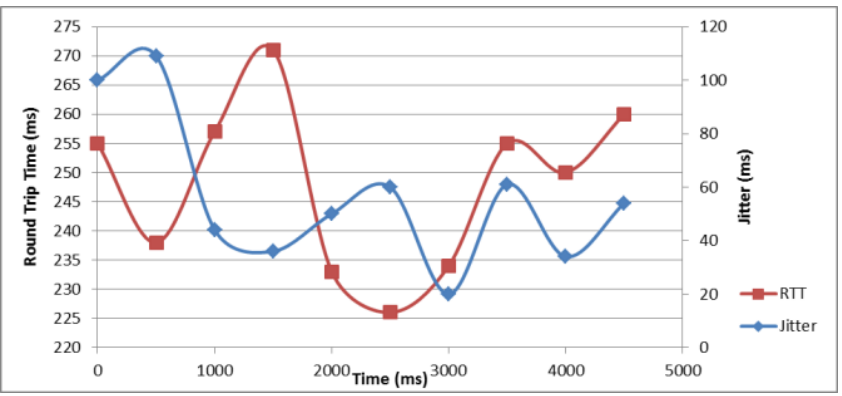

Figure 6. Round trip time vs jitter over a period of 4500 milliseconds of same country connections (Australia - Australia). 


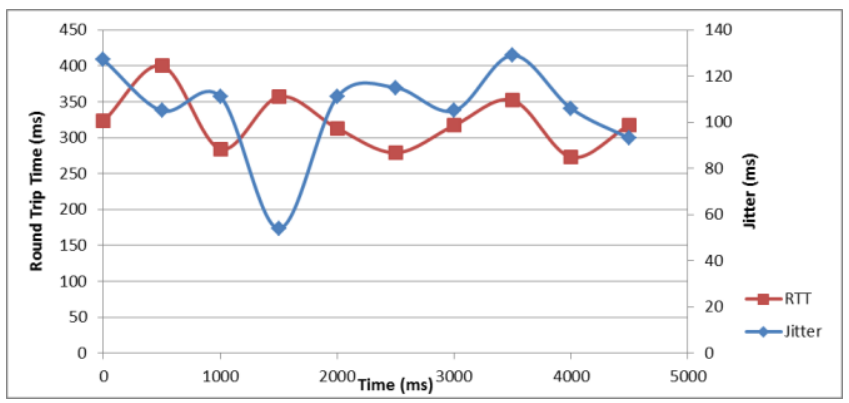

Figure 7. Round trip time vs jitter over a period of 4500 milliseconds of different country connections (Vietnam - Australia).

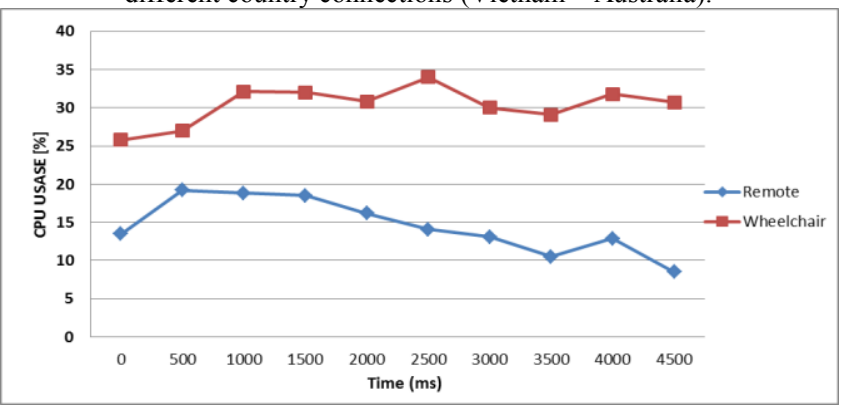

Figure 8. CPU usage of wheelchair computer and remote user's computer.

Through a series of careful measurements, the average RTT and jitter measurement results for local and global connections are shown in Fig. 6 and 7. It can be observed that round trip times fluctuated considerably between 226 and 271 milliseconds (ms) for local connections. RTTs of global connections were more than twice as high as that of local connections. In some cases, the global connection RTTs were in excess of $400 \mathrm{~ms}$. It is worth noting that the round trip time of less than $400 \mathrm{~ms}$ is considered as real-time videoconference [11]. In comparison with a similar previous study [12], our proposed system has superior performance.

Regarding the jitter of real-time traffic, there are significant differences between the local and international connections. The minimum jitter in the local connection is 20 $\mathrm{ms}$ and the minimum jitter in the international connection is under $60 \mathrm{~ms}$. Moreover, during our experiments, we also found that the performance of central processor unit (CPU) of the wheelchair computer and remote computer made a big different in terms of CPU usage.

Ultimately, all these parameters are correlated to the impact of mobility issues in system performance. There is a correlation also between the distance, radio reception strength, and delay. The data shows greater latency in long distance connections than in local connections. While these findings may not come as a surprise to most, it can be enhanced by implementing diversity network optimizations in the 4G networks. However, this study's RTT is still under the theoretical RTT threshold value [11]. It indicates that the latency impact of the cellular network is acceptable for remote users to control the wheelchair.

Our work demonstrates the successful experimental deployment of a telepresence wheelchair in an outdoor environment associated with a certain scenario. It was observed that in some cases the cellular network links disconnected and reconnected due to signal loss, radio interference, and cell re-selection. Nevertheless, the experimental results obtained have demonstrated the potential of a telepresence wheelchair using $4 \mathrm{G}$ cellular networks. Our future research will conduct experiments in the weak signal environments. The status of the wheelchair, the state of the wheelchair battery, the feedback information, the position tracking, and the user's biology signal would also be integrated. This system not only provides healthcare applications to wheelchair users but also acts as a bridge between wheelchair users, remote doctors, and their relatives. These positive outcomes may help the robotic and wheelchair community to make a significant investment in developing the telepresence wheelchair for healthcare.

\section{CONCLUSION}

We have presented the feasibility of the deployment of the telepresence wheelchair system in outdoor environments. The experimental results obtained on the wheelchair in a practical environment are positive and reliable, which shows potential for telepresence innovations in the health care domain. The results demonstrated the effectiveness of the combination of advanced technologies for a telepresence wheelchair. The system allows a remote user to control the wheelchair remotely and communicate with clients from a distant location through voice and video. Future work will consider transmitting feedback information and bio-signals to enable real-time health monitoring.

\section{REFERENCES}

[1] V. K. L. Ha, T. N. Nguyen, and H. T. Nguyen, "Real-time transmission of panoramic images for a telepresence wheelchair," in 37th Annual International Conference of the IEEE Engineering in Medicine and Biology Society (EMBC), 2015, pp. 3565-3568.

[2] A. V. Nguyen, L. B. Nguyen, S. Su, and H. T. Nguyen, "The advancement of an obstacle avoidance bayesian neural network for an intelligent wheelchair," in 35th Annual International Conference of the IEEE Engineering in Medicine and Biology Society (EMBC), 2013, pp. 3642-3645.

[3] J. S. Nguyen, N. Tuan Nghia, Y. Tran, S. W. Su, A. Craig, and H. T. Nguyen, "Real-time performance of a hands-free semi-autonomous wheelchair system using a combination of stereoscopic and spherical vision," in Annual International Conference of the IEEE Engineering in Medicine and Biology Society (EMBC), 2012, pp. 3069-3072.

[4] S. C. Herring, "Telepresence Robot for Academics," presented at the ASIST, Montreal, Quebec, Canada, 2013.

[5] K. Tatsuno, T. Kawai, M. Nako, Y. Yasuda, T. Fukuta, and H. Murata, "Development of a remote visitor robot system" in Annual Conference 2010, Proceedings of SICE, 2010, pp. 3616-3617.

[6] Microsoft Skype. (2015, 10 Oct). Available: http://www.skype.com

[7] G. Siwach and A. Esmailpour, "LTE Security potential vulnerability and algorithm enhancements," in 7th Canadian Conference on IEEE Electrical and Computer Engineering (CCECE), 2014, pp. 1-7.

[8] R. Kreher and K. Gaenger, LTE Signaling, Troubleshooting, and Optimization, John Wiley \& Sons, Ltd, 2010.

[9] A. Orebaugh, G. Ramirez, J. Burke, and L. Pesce, Wireshark $\mid \&$ Ethereal Network Protocol Analyzer Toolkit (Jay Beale's Open Source Security): Syngress Publishing, 2006.

[10]P. T. P. Ltd. (2014, 30 Jan). Mobile network guide improving mobile signal. Available: http://www.mobilenetworkguide.com.au

[11]ITU-T. Recommandation. G.1010, "Quality of service and performance", in Transmission systems and media, digital systems and networks, Geneva, Switzerland, 2001.

[12]J. D. Terrazas Gonzalez, G. Linton, F. Wai-keung, and R. Barwell, "On internet based supermedia enhanced telepresence via cellular data network," in 38th Annual Conference on IEEE Industrial Electronics Society IECON, 2012, pp. 2750-2755. 